\title{
Magnitude and Determinants of Antenatal Care Utilization in Kandahar City, Afghanistan
}

\author{
Muhammad Haroon Stanikzai $\left(\mathbb{D},{ }^{1}\right.$ Mohammad Hashim Wafa $\mathbb{D}^{2},{ }^{2}$ Abdul Wahed Wasiq, ${ }^{3}$ \\ and Hadia Sayam ${ }^{4}$ \\ ${ }^{1}$ Public Health Department, Faculty of Medicine, Kandahar University, Kandahar, Afghanistan \\ ${ }^{2}$ Neuropsychiatric and Behavioral Science Department, Faculty of Medicine, Kandahar University, Kandahar, Afghanistan \\ ${ }^{3}$ Internal Medicine Department, Faculty of Medicine, Kandahar University, Kandahar, Afghanistan \\ ${ }^{4}$ Para-Clinic Department, Faculty of Medicine, Malalay Institute of Higher Education, Kandahar, Afghanistan
}

Correspondence should be addressed to Muhammad Haroon Stanikzai; haroonstanikzai1@gmail.com

Received 15 May 2021; Accepted 28 June 2021; Published 3 July 2021

Academic Editor: Yoshitsugu Chigusa

Copyright ( 92021 Muhammad Haroon Stanikzai et al. This is an open access article distributed under the Creative Commons Attribution License, which permits unrestricted use, distribution, and reproduction in any medium, provided the original work is properly cited.

\begin{abstract}
Background. Women's and children's health is a crucial public health concern that epitomizes the universal platform for Sustainable Development Goals (SDGs). Appropriate and timely care during pregnancy can improve maternal and child health. Objectives. The present study aimed at determining the magnitude and determinants of antenatal care services' utilization in Kandahar city. Methods. A community-based cross-sectional study involving 850 women with at least one delivery in the last 2 years was carried out in Kandahar city from January to February 2021. Questionnaires to record information on sociodemographic, reproductive, and antenatal care- (ANC-) related characteristics were administered. Data were analyzed using SPSS 21.00 statistical software. We used descriptive statistics such as frequency and percentages to present the data. Determinants of antenatal care services' utilization were determined using a multivariable logistic regression model. Results. Among all study participants, $589(69.3 \%, 95 \%$ confidence interval $(\mathrm{CI})=66.0 \%-72.4 \%)$ of study participants utilized antenatal care services at least once. However, only $22 \%$ of the women were utilizing the recommended $\geq 4$ ANC visits. Factors that remained significantly associated with antenatal care services' utilization in multivariable analysis included women's educational status (adjusted odds ratio $(\mathrm{AOR})=2.0,95 \% \mathrm{CI}: 1.0-4.3)$, pregnancy intention $(\mathrm{AOR}=2.1,95 \% \mathrm{CI}: 1.1-3.4)$, and place of residence $(\mathrm{AOR}=1.7,95 \% \mathrm{CI}$ : 1.1-2.6). Conclusion. This study has found high rates (vs. the national level) of antenatal care services' utilization among women who had at least one delivery in the last 2 years. However, the rate of recommended $\geq 4$ ANC visits was low. Factors determining antenatal care utilization such as educational status of the mother, pregnancy intention, and place of residence hold the key to address the issue of ANC services lower utilization and consequently improve maternal and fetal health.
\end{abstract}

\section{Introduction}

Women's and children's health is a crucial public health concern that epitomizes the universal platform for Sustainable Development Goals (SDGs). Only in 2017, for instance, 295000 cases of maternal deaths were reported globally. It is asserted that maternal mortality figures pertain predominantly to low- and middle-income countries [1].

There is a myriad of well-established and cost-effective interventions, such as antenatal care (ANC), skilled attendant delivery, and postnatal care (PNC) that reduce maternal mortality. ANC entails specialized professional care offered to pregnant women from the onset of pregnancy until delivery. Its utilization ensures the prevention of maternal health risks, safe delivery, and good health of the newborn. It is therefore considered one of the four key strategies that connote reduction in maternal and childhood morbidity and mortality. World Health Organization (WHO) recommends a minimum of eight ANC contacts with the first visit during the first trimester of gestational age [2]. 
The proportion of women utilizing ANC services reflects discrepancies in different parts of the world. Moreover, ANC application is suboptimal, particularly in the developing world [3]. Data assert that about $98 \%$ of pregnant women receive ANC at least once in the developed world [3]. Of them, $81.9 \%$ initiate their ANC at the recommended time [4]. In the developing countries, however, it is as low as $68 \%$ (24\% early ANC) [4]. South Asia holds the lowest (54\%) ANC record of at least one ANC visit per pregnancy [3]. Therefore, it signifies a salient instance of a low-income region of the world. Likewise, Afghanistan Demographic Health Survey 2015 (ADHS, 2015) found that 59\% of pregnant women attended ANC visits at least once, while only $18 \%$ claimed four or more ANC visits $[5,6]$.

Perpetual findings of extensive research conducted in the developing world indicate that diverse factors such as maternal age $[7,8]$, maternal education $[6,8-10]$, household income $[8,10]$, husband education $[8,11]$, maternal employment $[12,13]$, area of residence $[8,10,14]$, pregnancy intention $[15,16]$, media exposure [16-18], pregnancylinked cost $[19,20]$, history of obstetric complications $[11,21]$, parity $[11,22]$, lack of ANC knowledge [23-25], and the distance from a health facility $[25,26]$ are associated with ANC utilization.

It is evident from ADHS (2015) that there was significant variation in antenatal care utilization by province. Women in the south were the lowest user of antenatal care services. Therefore, we sought to investigate the magnitude of antenatal care utilization and its determinants in Kandahar city. The present study would help policymakers to improve their knowledge regarding determinants of antenatal care utilization and subsequently can develop relevant policies for higher utilization of antenatal care services in the south.

\section{Materials and Methods}

2.1. Study Setting. This cross-sectional household survey was carried out in Kandahar city from January to February 2021. Kandahar is located in the southwest and is considered the second-largest city in Afghanistan. The city is divided into fifteen administrative units or districts and is lodging approximately 512000 people. In terms of public health service provision, there is a myriad of Primary Healthcare Clinics (PHCs), thirteen Comprehensive Health Clinics (CHCs), and two regional hospitals with secondary and tertiary healthcare capacities.

For data collection, we randomly selected four districts $(4,7,12,14)$ from the list of fifteen districts. A district typically lodges 7000-9000 households. The households within every district are numbered. The data were collected from 850 households.

2.2. Study Design. We employed a cross-sectional survey design, using quantitative data collection methods and statistical analysis. As this study is aimed at determining the determinants of ANC utilization, a cross-sectional design was appropriate because this design allows researchers to collect data on respondents' characteristics and information about the outcome simultaneously [18-22]. Furthermore, it determines the relationship between the independent characteristics of respondents with the outcome of interest.

2.3. Sample Size and Sampling Procedures. We employed single population proportion formula $\left[n=Z^{2} P(1-P) /(d)^{2}\right]$ for calculating our sample size. Our postulation of $95 \%$ confidence interval, $5 \%$ margin of error, $p=0.51$ [5] (the proportion of ANC services utilization in Kandahar), 10\% nonresponse rate, and a design effect of 2 resulted in a sample size of 850 households.

To pick a sample of households per district, we used stratified systematic random sampling. The number of selected households was proportionate to the household density of that district. The sampling interval $(k)$ for every district was extracted from the ratio of district households to the sample size. To select a starting household, we picked a random number from 1 to $k$, and thus every $k$ th household was included.

2.4. Inclusion and Exclusion Criteria. Participants with a history of at least one delivery in the last two years were eligible.

Unwillingness to participate and those who presented with a critical condition composed our exclusion criteria.

2.5. Study Variables. This study employed ANC service utilization status as a dependent variable. The outcome variable was binary and it was coded as 1 if the women were utilizing ANC services and 0 if the women were not utilizing ANC services. Our independent variables are well-documented and they consist of maternal age, educational level, employment status, residential area, income, pregnancy intention, history of obstetric complications, parity, birth order of the last child, and distance from health facility.

2.6. Data Collection. We used a structured and pretested questionnaire to collect the data. The study instruments were initially prepared in English and translated into Pashto and back into English to ensure that the meaning of questions is preserved. Prior to the commencement of the study, it was pilot tested on $5 \%$ of the total sample in another setting (Aino Mena, Kandahar city). The recruiters collected data pertinent to subjects' sociodemographic, reproductive, and ANC utilization status.

The data were collected by three pairs of nurses (one male and one female) and one supervisor (health professional). Prior to the pilot study the principal investigators organized a two-day training session for the recruiters. The objective was to enhance their skills of sampling, interviewing, filling out the questionnaires, and addressing potential ethical concerns that may emerge during the study. The quality of the collected data was assessed by the principal investigators through a random survey of the households. On a daily basis, the questionnaires were checked for completion. 
2.7. Statistical Analysis. All questionnaires were first coded and entered into Microsoft Excel (2019) and later exported into IBM SPSS version 21 for data cleaning and analysis [14]. We used a binary logistic regression model to assess the determinant of antennal natal care utilization. Variables with $p$ value of less than 0.25 were retained into multivariable logistic regression. Finally, multivariable logistics analysis with enter method was carried out to determine independent determinants of ANC utilization. The two-tailed $p$ values of $<0.05$ were considered statistically significant.

2.8. Ethical Consideration. This study received ethical clearance from the Research and Ethics Committee of Kandahar University (Makotob no. 53, dated on 28/7/2019). We obtained the permission of data collection from Kandahar Municipality. Additionally, the study was explained to every potential participant and informed verbal consents were obtained from all subjects.

\section{Results}

3.1. Sociodemographic Characteristics of the Subjects. The mean age of our subjects was $28( \pm 7.1)$. The vast majority of the subjects $(694,82 \%)$ were from the age group 20-35, 143 $(16.8 \%)$ were from the age group $>35$, and $13(1.5 \%)$ were from the age group $<20$. As portrayed in Table 1 , most of the respondents $(94.1 \%)$ had no formal education, $24(2.8 \%)$ had primary schooling, and only $18(2.1 \%)$ had attained religious education. Almost all (99.5\%) women were housewives and unemployed. Of their husbands' education and occupation, approximately $86.1 \%$ (732) of them had no formal education and about $93.3 \%$ (793) were self-employed. Moreover, $81.5 \%$ (693) of the subjects' families were living within $a<5 \mathrm{~km}$ distance of a health facility and more than half (64\%) of the families were having more than twelve members. In terms of their monthly household income, 516 (60.7\%) families of our subjects had a monthly household income of Afghanis $5000-10000$, and the rest $(334 ; 39.3 \%)$ gained $>$ Afghanis 10000 per month (Table 1 ).

3.2. Reproductive Characteristics of the Study Participants. We included a total of 850 women who delivered at least one viable child within the last two years. Of them, 770 (90.6\%) were multiparous. A minority of our subjects (158; $18.6 \%)$ have used diverse contraceptives before their last conception. Of them, $86(10.1 \%)$ used oral contraceptive pills and $44(5.2 \%)$ used male condoms. A staggering number of the subjects $(760 ; 89.4 \%)$ have planned their last pregnancies and $610(71.8 \%)$ have had experienced delivery in the hospital. Around $46.1 \%$ (392) of the women encountered complications during their last pregnancies. Table 2 depicts the characteristics of our subjects' reproductive variables.

3.3. Utilization of Antenatal Care Services. A total of 589 subjects $(69.3 \%, 95 \% \mathrm{CI}=66.07 \%-72.38 \%)$ utilized antenatal care services. Of these 336 (57\%, 95\% CI $=52.9 \%-$
TABLE 1: Sociodemographic characteristics of study participants $(n=850)$.

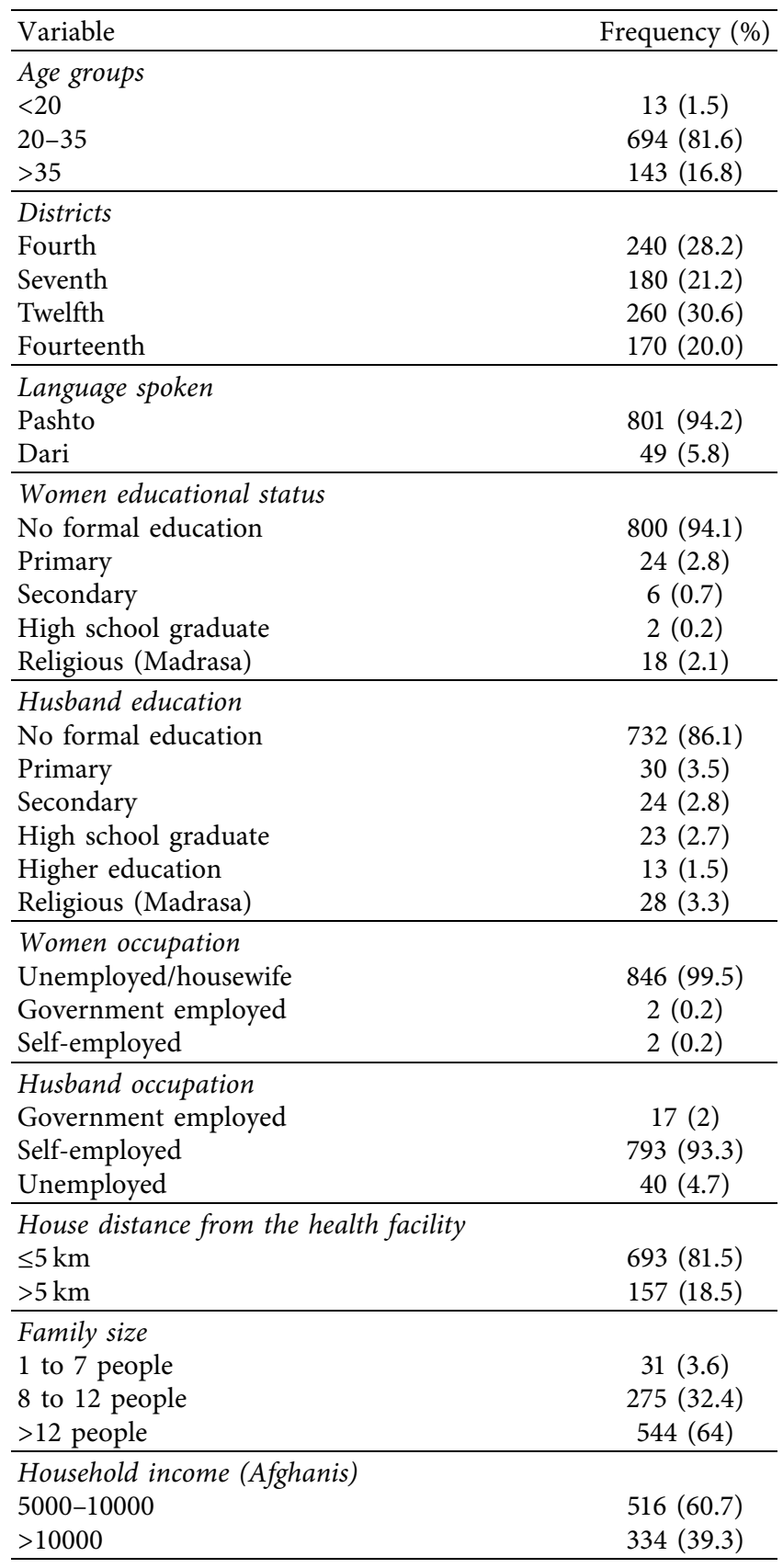

$61.08 \%)$ presented early (<12 weeks) and $253(43 \%, 95 \%$ $\mathrm{CI}=38.9 \%-47.06 \%)$ presented late $(>12$ weeks) for their first ANC visit. Our results reveal that only 128 (21.7\%) mothers asserted the recommended $(\geq 4)$ visits of the antenatal care services. Nearly 59\% (347) of our study participants visited Comprehensive Health Clinics (CHCs), 231 (39.3\%) visited hospitals, and $11(1.8 \%)$ visited private clinics for their antenatal care services. The majority of the subjects who utilized antenatal care services $(528 ; 89.6 \%)$ received health education in each ANC visit and 483 (82\%) believed that ANC is important for the health of both the mother and the child. Among 261 nonutilizers, common reasons for not 
TABLE 2: Reproductive characteristics of the study population.

\begin{tabular}{lc}
\hline Variable & Frequency (\%) \\
\hline Parity $(n=850)$ & $80(9.4)$ \\
Primiparous & $770(90.6)$ \\
Multiparous & \\
\hline Birth order of the last child $(n=850)$ & $509(59.9)$ \\
$1-3$ & $231(27.2)$ \\
$4-6$ & $110(12.9)$ \\
$\geq 7$ & \\
Contraceptive use in the past $(n=850)$ & $692(81.4)$ \\
Have not used & $44(5.2)$ \\
Male condom & $16(1.9)$ \\
Injectables & $86(10.1)$ \\
Oral contraceptives & $12(1.4)$ \\
IUD & \\
\hline Previous pregnancy planned $(n=850)$ & $760(89.4)$ \\
Yes & $90(10.6)$ \\
No & $610(71.8)$ \\
Place of last delivery $(n=850)$ & $240(28.2)$ \\
Health facility & $392(46.1)$ \\
Home & $458(53.9)$ \\
\hline History of complications in last pregnancy $(n=850)$ \\
Yes
\end{tabular}

utilizing ANC were family problems (125, 47.9\%), transportation problems $(67,25.7 \%)$, and lack of awareness (57, 21.8\%) (Table 3).

\subsection{Determinants of Antenatal Care Services' Utilization.} The multiple logistic regression showed that the odds of utilizing antenatal care services were higher in educated women $(\mathrm{AOR}=2.09,95 \% \mathrm{CI}: 1.00-4.37)$ as compared to women with no formal education. Besides, women who planned their last pregnancy had 2.16 times higher odds of utilizing antenatal care services $(\mathrm{AOR}=2.16,95 \% \mathrm{CI}$ : 1.14-3.40) than the women with an unplanned pregnancy. Furthermore, in contrast to the women living in the twelfth district the expected number of antenatal care utilization visits for the women who were living in the seventh district was 1.72 times higher $(\mathrm{AOR}=1.72,95 \% \mathrm{CI}$ : 1.10-2.67). Table 4 summarizes the results of the bivariate and multivariable analysis.

\section{Discussion}

This is the first study, of which we are aware, to determine the magnitude of ANC services utilization and its determinants among women who had at least one delivery in the last two years in Kandahar city. The results of this community-based cross-sectional study were consistent with the findings of other pertinent studies conducted in developing countries [7-11, 18, 23]. We found that ANC utilization was highly associated with the educational status of the mother, pregnancy intention, and her place of residence. Our findings showed that these factors should be considered in future programs to enhance the utilization of antenatal care services.
TABLE 3: Antenatal care utilization and its related characteristics.

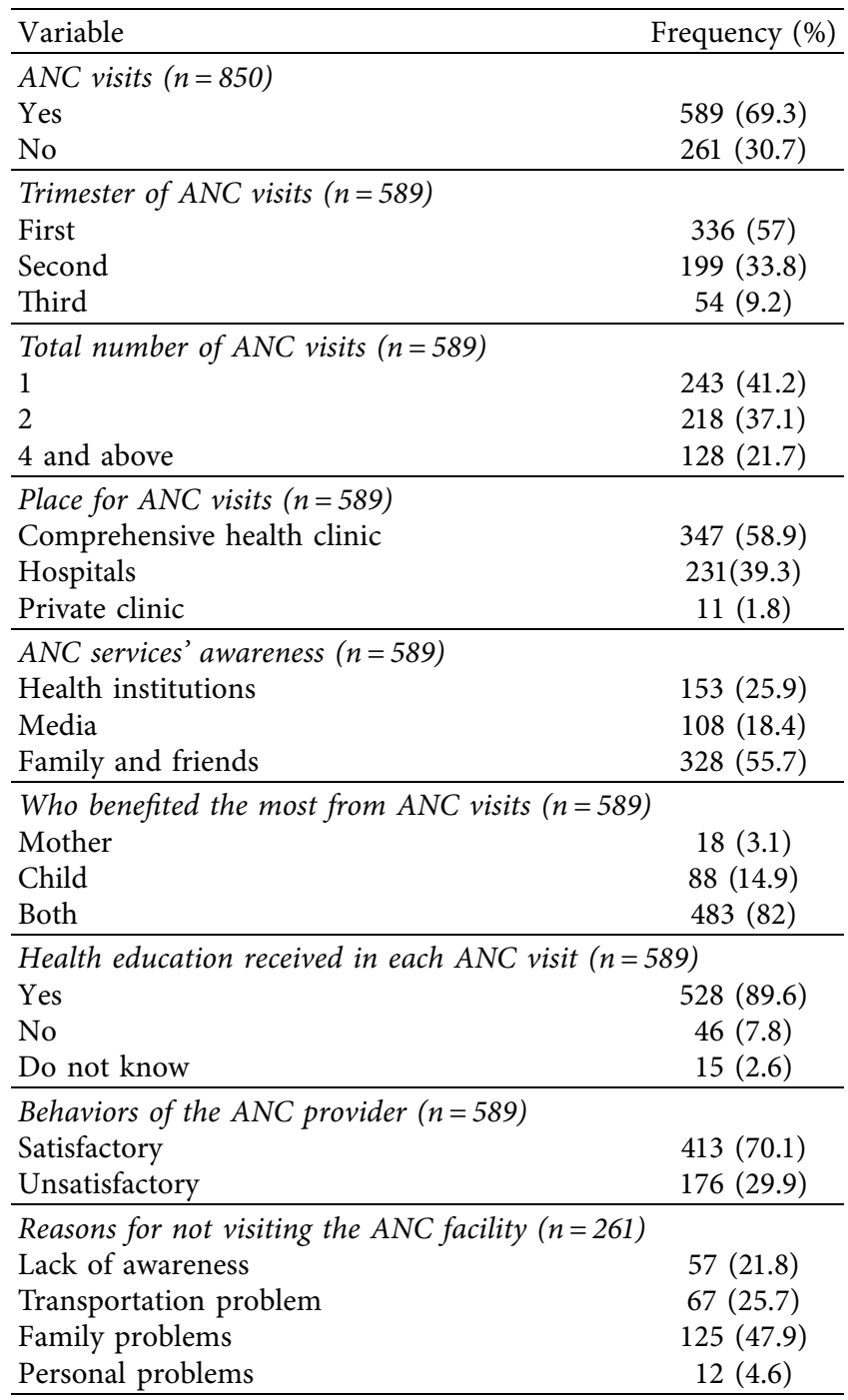

We found that $69.3 \%$ of our subjects (the mothers) made at least one ANC visit with a $95 \%$ CI of $66.07 \%$ to $72.38 \%$. This figure is higher than the national level (59\%) reported by ADHS in $2015[5,6]$ and comparable with another study conducted in Kandahar province [27]. The rate of ANC utilization in the present study is consistent with those asserted in many studies conducted in other developing countries, such as Rwanda, Bangladesh, Jordan, and Ethiopia [7-11, 18, 23]. This finding epitomizes many developing countries where a substantial proportion of pregnant women fail to regularly make their ANC visits. ANC utilization rates, however, have been shown higher in the developed world probably due to greater access and awareness as well as other sociodemographic disparities between the two settings [4].

We noticed that out of 589 mothers who did visit ANC services only $22 \%$ of them have made the recommended $\geq 4$ ANC visits. This finding of low utilization is of great importance. It suggests that while a significant number of the mothers (69.3\%) were aware of ANC services, a vast majority did not succeed to make the recommended $\geq 4$ ANC visits. 
TABLE 4: Determinants of antenatal care utilization in Kandahar city, 2021: crude and adjusted odds ratio.

\begin{tabular}{|c|c|c|c|c|c|}
\hline Independent variable & Categories & Crude odds ratio $(95 \% \mathrm{CI})$ & $p$ value & Adjusted odds ratio $(95 \% \mathrm{CI})$ & $p$ value \\
\hline $\begin{array}{l}\text { Women } \\
\text { education }\end{array}$ & $\begin{array}{c}\text { Educated } \\
\text { Uneducated }\end{array}$ & $\begin{array}{c}3.09(2.00-4.37) \\
1\end{array}$ & 0.045 & $\begin{array}{c}2.09(1.00-4.37) \\
1\end{array}$ & 0.017 \\
\hline Previous pregnancy & $\begin{array}{c}\text { Planned } \\
\text { Unplanned }\end{array}$ & $\begin{array}{c}2.16(1.38-3.36) \\
1 \\
\end{array}$ & 0.001 & $\begin{array}{c}2.10(1.14-3.40) \\
1 \\
\end{array}$ & 0.014 \\
\hline District & $\begin{array}{l}\text { Twelve } \\
\text { Seven } \\
\text { Four } \\
\text { Fourteen }\end{array}$ & $\begin{array}{c}1 \\
1.92(1.24-2.95) \\
1.22(0.84-1.77) \\
0.37(0.48-1.11)\end{array}$ & $\begin{array}{c}0.003 \\
0.29 \\
0.14\end{array}$ & $\begin{array}{c}1 \\
1.72(1.10-2.67) \\
- \\
-\end{array}$ & 0.017 \\
\hline
\end{tabular}

Hence, further studies are warranted to address what brings about such low utilization of ANC services.

Our data indicate a fairly strong association between women' educational status and the utilization of ANC services. Women who had basic, secondary, and tertiary education were more likely to utilize ANC services in comparison to those who had no formal education. This finding is consistent with previous studies from other developing countries $[6,8-10]$. One solution is to strengthen and expand primary education and female literacy programs to improve women's health in the long term [5]. Other approaches include health educational programs and public awareness campaigns to be undertaken in the light of low health literacy on the utilization of antenatal care services in Kandahar province.

Additionally, we observed that pregnancy intention was a significant determinant of ANC services utilization. Women with planned pregnancies were about two times more likely to utilize ANC than those with unplanned pregnancies. Studies conducted in Ethiopia, Brazil, India, and other developing countries reflect a comparable finding $[15,28-30]$. Given this finding, it is imperative to increase the uptake of family planning services that will promote planned pregnancies and can subsequently encourage the utilization of antenatal care services [31].

As our third important finding, and consistent with other studies conducted in Bangladesh [8] and Ethiopia [10], the area of residence of the mothers was strongly associated with ANC utilization; e.g., women living in the seventh district of Kandahar city were about two times more likely to utilize ANC services than those who lived in the twelfth district. This higher rate of ANC utilization among the residents of the seventh district probably reflects the value of living in the close vicinity of a health facility. It is also argued that there might be other system-related factors, such as home visits and more health facilities, that may have influenced ANC services utilization in this district. Hence, addressing the aforementioned barriers holds the key to improving antenatal care utilization in these settings.

\section{Strengths and Limitations}

Although this study found some crucial factors that were significantly associated with ANC utilization by pregnant women, our findings are to be interpreted in light of the following limitations. First, the cross-sectional nature of the study limits the temporal relationship between the variables. Secondly, insecurity of our study area and the poverty of the subjects may have somehow influenced our results. Furthermore, we defined place of residence as the respondent's residential area during the data collection period, but antenatal care utilization was assessed over the last two years and therefore there might be a measurement bias. Finally, our data were collected in the metropolitan area. Therefore, its generalizability is limited in terms of not representing the rural population and nomads. We suggest that further in-depth investigation of health system-related factors associated with ANC utilization is highly needed to be conducted on provincial and country levels.

\section{Conclusion}

In this community-based study, we found that more than half $(69.3 \%)$ of our subjects utilized ANC services at least once. However, only $22 \%$ of the women were utilizing the recommended $\geq 4$ ANC visits. Factors determining antenatal care utilization such as educational status of the mother, pregnancy intention, and place of residence hold the key to address the issue of ANC services lower utilization and consequently improve maternal and fetal health.

\section{Implications for Practice and/or Policy}

This study provides information on the determinants of antenatal care utilization in Kandahar city. Policymakers and program planners should be aware that factors such as the education status of the women, pregnancy intention, and place of residence are associated with a greater chance of ANC utilization. Limited information is available on the health-system-related factors that influence ANC utilization. We recommend further in-depth investigation of health system-related factors associated with ANC utilization on provincial and country levels.

\section{Data Availability}

The primary data used to support the findings of this study are available from the corresponding author upon request.

\section{Conflicts of Interest}

The authors declare no conflicts of interest. 


\section{Authors' Contributions}

MHS, MHW, and AWW conceptualized and designed the study. HS and MHS performed analysis. MHS, MHW, and HS wrote the original draft. MHW, MHS, HS, and AWW edited and reviewed the article. All authors read and approved the final manuscript.

\section{Acknowledgments}

The authors express their gratitude to the leaders and administrative bodies of the districts. Their special thanks go to the data collectors and respondents of this study whose contribution made this study possible.

\section{References}

[1] J. Bongaarts, "WHO, UNICEF, UNFPA, World Bank Group, and United Nations population division trends in maternal mortality: 1990 to 2015," Population and Development Review, vol. 42, no. 4, 726 pages, 2016.

[2] M. Uzun Aksoy and E. Gürsoy, "World health organization recommendations on antenatal care for a positive pregnancy experience," International Refereed Journal of Gynecology and Maternal Child Health, WHO, Geneva, Switzerland, 2016.

[3] Antenatal Care in Developing Countries Promises, Achievements and Missed Opportunities. 2020: http://www. unicef.org/media/files/antenatal.pdf.

[4] A.-B. Moller, M. Petzold, D. Chou, and L. Say, "Early antenatal care visit: a systematic analysis of regional and global levels and trends of coverage from 1990 to 2013," The Lancet Global Health, vol. 5, no. 10, pp. e977-e983, 2017.

[5] Central Statistics Organization/Afghanistan, Ministry of Public Health/Afghanistan, and ICF. 2017. Afghanistan Demographic and Health Survey 2015. Kabul, Afghanistan: Central Statistics Organization, http://dhsprogram.com/ pubs/pdf/FR323/FR323.pdf.x.

[6] S. Mumtaz, J. Bahk, and Y. H. Khang, "Current status and determinants of maternal healthcare utilization in Afghanistan: analysis from Afghanistan demographic and health survey 2015," PLoS One, vol. 14, no. 6, Article ID e0217827, 2019.

[7] A. A. Rurangirwa, I. Mogren, L. Nyirazinyoye, J. Ntaganira, and G. Krantz, "Determinants of poor utilization of antenatal care services among recently delivered women in Rwanda; a population based study," BMC Pregnancy and Childbirth, vol. 17, no. 1, 2017.

[8] S. K. Chanda, B. Ahammed, M. H. Howlader, M. Ashikuzzaman, T.-E.-A. Shovo, and M. T. Hossain, "Factors associating different antenatal care contacts of women: a cross-sectional analysis of Bangladesh demographic and health survey 2014 data," PLoS One, vol. 15, no. 4, Article ID e0232257, 2020.

[9] H. H. Hijazi, M. S. Alyahya, A. M. Sindiani, R. S. Saqan, and A. M. Okour, "Determinants of antenatal care attendance among women residing in highly disadvantaged communities in northern Jordan: a cross-sectional study," Reproductive Health, vol. 15, no. 1, 106 pages, 2018.

[10] K. Tiruaynet and K. F. Muchie, "Determinants of utilization of antenatal care services in Benishangul Gumuz Region, Western Ethiopia: a study based on demographic and health survey," BMC Pregnancy and Childbirth, vol. 19, no. 1, 2019.
[11] I. N. Okedo-Alex, I. C. Akamike, O. B. Ezeanosike, and C. J. Uneke, "Determinants of antenatal care utilisation in Sub-Saharan Africa: a systematic review," BMJ Open, vol. 9, no. 10, Article ID e031890, 2019.

[12] B. K. Sarker, M. Rahman, T. Rahman et al., "Status of the WHO recommended timing and frequency of antenatal care visits in Northern Bangladesh," PLoS One, vol. 15, no. 11, Article ID e0241185, 2020.

[13] S. Sharma, P. Sarathi Mohanty, R. Omar, A. P. Viramgami, and N. Sharma, "Determinants and utilization of maternal health care services in urban slums of an industrialized city, in western India," Journal of Family \& Reproductive Health, vol. 14, 2020.

[14] T. Mekonnen, T. Dune, J. Perz, and F. A. Ogbo, “Trends and determinants of antenatal care service use in Ethiopia between 2000 and 2016," International Journal of Environmental Research and Public Health, vol. 16, no. 5, 748 pages, 2019.

[15] Y. Dibaba, M. Fantahun, and M. J. Hindin, "The effects of pregnancy intention on the use of antenatal care services: systematic review and meta-analysis," Reproductive Health, vol. 10, no. 1, 50 pages, 2013.

[16] K. Birmeta, Y. Dibaba, and D. Woldeyohannes, "Determinants of maternal health care utilization in Holeta town, central Ethiopia," BMC Health Services Research, vol. 13, no. 1, 256 pages, 2013.

[17] S. K. Ousman, I. Mdala, V. C. Thorsen, J. Sundby, and J. H. Magnus, "Social determinants of antenatal care service use in Ethiopia: changes over a 15-year span," Frontiers in Public Health, vol. 7, 2019.

[18] S. Saha, M. Mubarak, and J. Jarl, "What socioeconomic factors are associated with different levels of antenatal care visits in Bangladesh? A behavioral model," Health Care for Women International, vol. 38, no. 1, pp. 2-16, 2016.

[19] M. Vidler, U. Charantimath, G. Katageri et al., "Community perceptions of pre-eclampsia in rural Karnataka State, India: a qualitative study," Reproductive Health, vol. 13, no. S1, 2016.

[20] G. T. Nachinab, C. A. Adjei, F. A. Ziba, R. Asamoah, and P. A. Attafuah, "Exploring the determinants of antenatal care services uptake: a qualitative study among women in a rural community in northern Ghana," Journal of Pregnancy, vol. 2019, Article ID 3532749, 6 pages, 2019.

[21] N. Moore, B. Blouin, H. Razuri, M. Casapia, and T. W. Gyorkos, "Determinants of first trimester attendance at antenatal care clinics in the Amazon region of Peru: a casecontrol study," PLoS One, vol. 12, no. 2, Article ID e0171136, 201716.

[22] T. Dahiru and O. M. Oche, "Determinants of antenatal care, institutional delivery and postnatal care services utilization in Nigeria," Pan African Medical Journal, vol. 21, 2015.

[23] S. Aziz Ali, A. Feroz, S. Saleem, Z. Fatmai, and M. M. Kadir, "Factors affecting the utilization of antenatal care among married women of reproductive age in the rural Thatta, Pakistan: findings from a community-based case-control study," BMC Pregnancy and Childbirth, vol. 20, no. 1, 2020.

[24] C. Wilunda, G. Quaglio, G. Putoto et al., "Determinants of utilization of antenatal care and skilled birth attendant at delivery in South West Shoa Zone, Ethiopia: a cross sectional study," Reproductive Health, vol. 12, no. 1, 2015.

[25] Y. A. Bekele, T. E. Tafere, A. A. Emiru, and H. B. Netsere, "Determinants of antenatal care dropout among mothers who gave birth in the last six months in BAHIR Dar ZURIA WOREDA community; mixed designs," BMC Health Services Research, vol. 20, no. 1, 2020. 
[26] S. Lakew, A. Ankala, and F. Jemal, "Determinants of client satisfaction to skilled antenatal care services at Southwest of Ethiopia: a cross-sectional facility based survey," $B M C$ Pregnancy and Childbirth, vol. 18, no. 1, 479 pages, 2018.

[27] M. Saheem, M. H. Stanikzai, N. Rahimy, N. Fazli, G. M. Mudasir, and H. Sayam, "Factors associated with modern contraceptive use among married women attending comprehensive health centers (CHCs) in kandahar," International Journal of Reproductive Medicine, vol. 2021, Article ID 6688459, 7 pages, 2021.

[28] T. Tekelab, C. Chojenta, R. Smith, and D. Loxton, "Factors affecting utilization of antenatal care in Ethiopia: a systematic review and meta-analysis," PLoS One, vol. 14, no. 4, Article ID e0214848, 2019.

[29] D. G. Bassani, P. J. Surkan, and M. T. A. Olinto, "Inadequate use of prenatal services among Brazilian women: the role of maternal characteristics," International Perspectives on Sexual and Reproductive Health, vol. 35, no. 1, pp. 15-20, 2009.

[30] G. Kumar, T. S. Choudhary, A. Srivastava et al., "Utilisation, equity and determinants of full antenatal care in India: analysis from the national family health survey 4 ," $B M C$ Pregnancy and Childbirth, vol. 19, no. 1, 2019.

[31] A. O. Tsui, R. McDonald-Mosley, and A. E. Burke, "Family planning and the burden of unintended pregnancies," Epidemiologic Reviews, vol. 32, no. 1, pp. 152-174, 2010. 